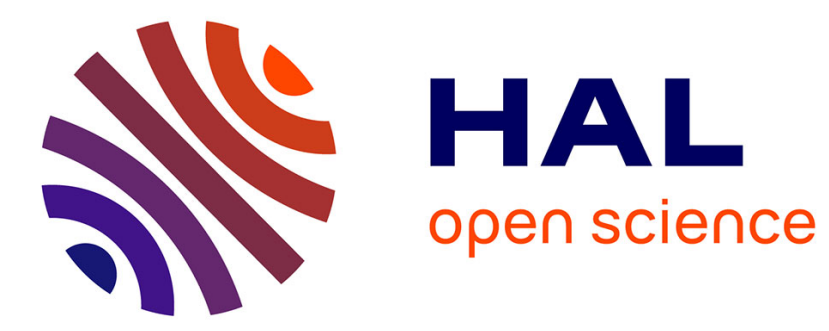

\title{
Stability and Stabilization of Homogeneous Systems Depending on a Parameter \\ Emmanuel Moulay
}

\section{To cite this version:}

Emmanuel Moulay. Stability and Stabilization of Homogeneous Systems Depending on a Parameter. IEEE Transactions on Automatic Control, 2009, 54 (6), pp.1382-1385. 10.1109/TAC.2009.2015560 . hal-03088812

\section{HAL Id: hal-03088812 https://hal.science/hal-03088812}

Submitted on 19 Jan 2021

HAL is a multi-disciplinary open access archive for the deposit and dissemination of scientific research documents, whether they are published or not. The documents may come from teaching and research institutions in France or abroad, or from public or private research centers.
L'archive ouverte pluridisciplinaire HAL, est destinée au dépôt et à la diffusion de documents scientifiques de niveau recherche, publiés ou non, émanant des établissements d'enseignement et de recherche français ou étrangers, des laboratoires publics ou privés. 


\title{
Stability and stabilization of homogeneous systems depending on a parameter
}

\author{
Emmanuel Moulay
}

\begin{abstract}
This paper deals with the Lyapunov stability and stabilization of nonlinear homogeneous systems depending on a parameter. We give a new method for proving global stability and stabilization by using a continuous topological deformation which preserves the stability. The proposed results use a condition based on a well known topological lemma, called the tube lemma. To illustrate these results, we study finite time robust stabilization of the chain of integrators.
\end{abstract}

Index Terms-Homogeneous systems, nonlinear systems depending on a parameter, stability, stabilization.

\section{INTRODUCTION}

The stabilization of nonlinear systems is a challenging and difficult subject in control theory. Recent years have seen increasing interest in the stabilization of nonlinear systems. There are several motivations for considering homogeneous systems depending continuously on a parameter. The main one is that it can be seen as a continuous topological deformation which preserves the stability. The proposed framework is based on a topological result known as the tube lemma and leads to a new method for proving global stability and stabilization of homogeneous systems. This idea has been considered in [1] to study the finite time stabilization of the chain of integrators. The main objective of this paper is to extend the scope of the study to the global stability and stabilization of homogeneous systems depending on a parameter.

One system of particular interest in many practical situations is the chain of integrators. We apply our theoretical results to the finite time robust stabilization of this chain of integrators. A survey on finite time stability of nonlinear systems can be found in [2] and the problem of finite stabilization of nonlinear systems is considered for instance in [3].

The outline of the paper is as follows. In Section II, we fix notation and terminology about control theory. Our main result for the stability and the stabilization of systems depending on a parameter is proved in Section III. In Section IV we discuss the implications of this result to the finite time robust stabilization of the chain of integrators. We make concluding remarks and sketch some directions for future research in Section V.

\section{NOTATION AND DEFINITIONS}

Throughout the paper $\mathcal{B}^{n}$ denotes the unit open ball of $\mathbb{R}^{n}$. A neighborhood of $x$ in a topological space $X$ is an open subset $U$ of $X$ which contains $x$. Let $A$ be a subset of a topological space, $\partial A$ and $\AA$ denote the boundary and the

E. Moulay is with IRCCyN (UMR-CNRS 6597), 1 rue de la Noë, B.P. 92 101, 44321 Nantes CEDEX 03, France (e-mail: Emmanuel.Moulay@ irccyn.ec-nantes.fr) interior of $A$. A function $f: \mathbb{R}^{n} \rightarrow \mathbb{R}^{m}$ is a smooth function if it is at least $C^{1}$.

A matrix $A \in \mathbb{C}^{n \times n}$ is Hurwitz if its eigenvalues have strictly negative real part. We denote by

$$
C(\alpha)=\left[\begin{array}{ccccc}
0 & 0 & \cdots & 0 & \alpha_{1} \\
1 & 0 & \cdots & 0 & \alpha_{2} \\
0 & 1 & \cdots & 0 & \alpha_{3} \\
\vdots & \vdots & \ddots & \vdots & \vdots \\
0 & 0 & \cdots & 1 & \alpha_{n}
\end{array}\right]
$$

the companion matrix attached to $\alpha=\left(\alpha_{1}, \ldots, \alpha_{n}\right) \in \mathbb{C}^{n}$.

A function $g: X \rightarrow Y$ between two topological spaces is proper if and only if the preimage of every compact set in $Y$ is compact in $X$.

Let us recall the definitions about weighted homogeneity which generalizes the classical notion of homogeneity and has been first introduced in [4]. A function $V: \mathbb{R}^{n} \rightarrow \mathbb{R}$ is $r$-homogeneous of degree $d$ if

$$
V\left(\Delta_{\lambda}^{r}(x)\right)=\lambda^{d} V(x)
$$

for all $\lambda>0$ where $r=\left(r_{1}, \ldots, r_{n}\right) \in \mathbb{R}_{>0}^{n}$ are the weights and $\Delta_{\lambda}^{r}(x)=\left(\lambda^{r_{1}} x_{1}, \ldots, \lambda^{r_{n}} x_{n}\right)$ is the dilatation. A vector field $f$ is $r$-homogeneous of degree $d$ if for all $1 \leq i \leq n$, the $i$-th component $f_{i}$ is a $r$-homogeneous function of degree $r_{i}+d$, that is

$$
f_{i}\left(\Delta_{\lambda}^{r}(x)\right)=\lambda^{r_{i}+d} f_{i}(x)
$$

for all $\lambda>0$.

Let us consider a continuous vector field $f: \mathbb{R}^{n} \rightarrow \mathbb{R}^{n}$. In the following, we consider that all Cauchy problems associated with the system

$$
\dot{x}=f(x), \quad x \in \mathbb{R}^{n}
$$

have unique forward solutions for all positive times denoted by $\phi^{x}(t)$ for $x \in \mathbb{R}^{n}$ and $t \geq 0$. Under this assumption on $f$, the system (1) has a continuous global semi-flow $\psi(t, x)=\phi^{x}(t)$ (see [5]). A set $A \subseteq \mathbb{R}^{n}$ is positively invariant under $f$ if $\psi(t, A) \subseteq A$ for all $t>0$. A set $A$ that is positively invariant under $f$ is strictly positively invariant under $f$ if $\psi(t, A) \subset \AA$ for all $t>0$.

The system (1) is $r$-homogeneous of degree $d$ if the vector field $f$ is $r$-homogeneous of degree $d$.

If $V: \mathbb{R}^{n} \rightarrow \mathbb{R}$ is a continuously differentiable function then define

$$
\dot{V}(x)=\sum_{i=1}^{n} \frac{\partial V}{\partial x_{i}}(x) f_{i}(x) .
$$


A Lyapunov function $V: \mathbb{R}^{n} \rightarrow \mathbb{R}_{\geq 0}$ for the system (1) is a proper continuously differentiable positive definite function such that $\dot{V}$ is negative definite.

The system (1) is asymptotically stable if the following two conditions hold.

1) the system (1) is stable, that is, for every open neighborhood $\mathcal{V} \subseteq \mathbb{R}^{n}$ of the origin, there exists an open neighborhood $\mathcal{U} \subseteq \mathbb{R}^{n}$ of the origin such that $\psi(t, \mathcal{U}) \subseteq \mathcal{V}$ for every $t \geq 0$.

2) the origin is attractive, that is, there exists an open neighborhood $\mathcal{W} \subseteq \mathbb{R}^{n}$ of the origin such that, for every $x \in \mathcal{W}$ and every open neighborhood $\mathcal{U} \subseteq \mathbb{R}^{n}$ of the origin, there exists $T \geq 0$ such that $\psi(t, x) \in \mathcal{U}$ for all $t>T$.

The system (1) is globally asymptotically stable if it is asymptotically stable on the whole space $\mathcal{W}=\mathbb{R}^{n}$.

Let us introduce the notion of finite time stability involving the settling time function given in [6].

Definition 1: Let us consider the system (1). The system (1) is finite time stable if it is stable and there exists an open neighborhood $\mathcal{N}$ of the origin that is positively invariant under $f$ and a positive definite function $T: \mathcal{N} \rightarrow \mathbb{R}$ called the settling-time function such that $\psi(T(x), x)=0$ for all $x \in \mathcal{N}$ and $\psi(t, x) \neq 0$ for all $x \in \mathcal{N} \backslash\{0\}, t<T(x)$. The system (1) is globally finite time stable if the it is finite time stable with $\mathcal{N}=\mathbb{R}^{n}$.

Various properties of the settling-time function are given in [6].

Remark 2: If the system (1) is finite time stable, then it cannot possess uniqueness in backward time at the origin, in particular $f$ cannot be locally Lipschitz at the origin.

Consider the control system

$$
\dot{x}=f(x, u), \quad x \in \mathbb{R}^{n}, u \in \mathbb{R}^{m}
$$

with $f \in C^{0}\left(\mathbb{R}^{n} \times \mathbb{R}^{m}, \mathbb{R}^{n}\right)$ and $f(0,0)=0$.

The control system (2) is continuously stabilizable (respectively globally continuously stabilizable) if there exists a feedback control $u \in C^{0}\left(\mathbb{R}^{n}, \mathbb{R}^{m}\right)$ such that:

1) $u(0)=0$,

2) the closed-loop system

$$
\dot{x}=f(x, u(x)), \quad x \in \mathbb{R}^{n} .
$$

is asymptotically stable (respectively globally asymptotically stable).

The control system (2) is finite time stabilizable (respectively globally finite time stabilizable) if the closed-loop system (3) is finite time stable (respectively globally finite time stable).

\section{STABILITY AND STABILIZATION OF SYSTEMS DEPENDING ON A PARAMETER}

For the convenience of the reader, we first recall a result dedicated to homogeneous vector fields which can be found in [1, Theorem 6.1].

Lemma 3: Suppose that the vector field $f$ is homogeneous. If $\mathcal{A} \subset \mathbb{R}^{n}$ is a compact set that contains the origin and is strictly positively invariant under $f$, then the system (1) is globally asymptotically stable.

Let us recall a Lyapunov theorem dedicated to the homogeneous systems which appears in [7, Theorem 2].

Lemma 4: Suppose that the vector field $f$ is continuous on $\mathbb{R}^{n}$ and $r$-homogeneous of degree $d$. Let $p$ be a positive integer. If the system (1) is asymptotically stable, then there exists a $C^{p}$-Lyapunov function for the system (1) $r$-homogeneous of degree

$$
k>p \max _{1 \leq i \leq n} r_{i} .
$$

Now, let us consider a continuous function

$$
f: \Lambda \times \mathbb{R}^{n} \rightarrow \mathbb{R}^{n}
$$

where $\Lambda$ is a topological space, and for $\omega \in \Lambda$ the system

$$
\dot{x}=f(\omega, x), \quad x \in \mathbb{R}^{n} .
$$

For every $\omega \in \Lambda$, we suppose in the following that all Cauchy problems associated with the system (4) have unique forward solutions for all positive times.

The following theory is based on a well known topological lemma called the tube lemma. Let us recall this lemma whose proof can be found in [8, Lemma 26.8].

Lemma 5 (tube lemma): Let $X$ and $Y$ be topological spaces with $Y$ compact and consider the product space $X \times Y$. If $N$ is an open set of $X \times Y$ containing the slice $\left\{x_{0}\right\} \times Y$ of $X \times Y$, then $N$ contains some tube $W \times Y$ about $\left\{x_{0}\right\} \times Y$, where $W$ is a neighborhood of $x_{0}$ in $X$.

We can now state the main result of this paper.

Theorem 6: Let $\Lambda$ be a topological space and suppose that $f$ is a continuous vector field on $\Lambda \times \mathbb{R}^{n}$ such that $x \mapsto f(\omega, x)$ is $\left(r_{1}(\omega), \ldots, r_{n}(\omega)\right)$-homogeneous ${ }^{1} \in \mathbb{R}_{>0}^{n}$. If the system

$$
\dot{x}=f\left(\omega_{0}, x\right), \quad x \in \mathbb{R}^{n}
$$

is globally asymptotically stable for $\omega_{0} \in \Lambda$, then there exist a neighborhood $\Lambda^{\prime}$ of $\omega_{0}$ in $\Lambda$ such that for all $\omega \in \Lambda^{\prime}$, the system (4) is globally asymptotically stable. Moreover, if the weights $r=\left(r_{1}, \ldots, r_{n}\right) \in \mathbb{R}_{>0}^{n}$ do not depend on $\omega$, then for any $r$-homogeneous smooth Lyapunov function $V$ for the system (5) there exists a neighborhood $\Lambda^{\prime \prime}$ of $\omega_{0}$ in $\Lambda$ such that $V$ is also a Lyapunov function for the system (4) for all $\omega \in \Lambda^{\prime \prime}$.

Proof: As the system (5) is globally asymptotically stable, the theorem of Kurzweil given in [9, Theorem 7] ensures there exists a smooth Lyapunov function $V: \mathbb{R}^{n} \rightarrow \mathbb{R}_{\geq 0}$ for the system (5). Let us denote by

$$
\dot{V}_{\omega}(x)=\sum_{i=1}^{n} \frac{\partial V}{\partial x_{i}}(x) f_{i}(\omega, x) .
$$

As $V$ is proper,

$$
\mathcal{S}=V^{-1}(\{1\})
$$

is compact. Define the continuous function

$$
\begin{aligned}
\varphi: \Lambda \times \mathcal{S} & \rightarrow \mathbb{R} \\
(\omega, x) & \mapsto \dot{V}_{\omega}(x)
\end{aligned}
$$

\footnotetext{
${ }^{1}$ In general, the weights $\left(r_{1}(\omega), \ldots, r_{n}(\omega)\right)$ depend on $\omega$.
} 
then $\varphi^{-1}\left(\mathbb{R}_{<0}\right)$ is an open subset of $\Lambda \times \mathcal{S}$ containing the slice $\left\{\omega_{0}\right\} \times \mathcal{S}$. Since $\mathcal{S}$ is compact, there exists a neighborhood $\Lambda^{\prime} \subseteq \Lambda$ of $\omega_{0}$ such that $\varphi^{-1}\left(\mathbb{R}_{<0}\right)$ contains some tube $\Lambda^{\prime} \times \mathcal{S}$ about $\left\{\omega_{0}\right\} \times \mathcal{S}$ (see Lemma 5). We have $\dot{V}_{\omega}(x)<0$ for all $(\omega, x) \in \Lambda^{\prime} \times \mathcal{S}$. The compact set

$$
\mathcal{A}=V^{-1}([0,1])
$$

is strictly positively invariant under $x \mapsto f(\omega, x)$ for all $\omega$ in $\Lambda^{\prime}$, due to the fact that

$$
\mathcal{S}=\partial \mathcal{A}
$$

By using Lemma 3, we deduce that the system (4) is globally asymptotically stable for every $\omega$ in $\Lambda^{\prime}$.

Now, suppose that the weights $r=\left(r_{1}, \ldots, r_{n}\right) \in \mathbb{R}^{n}$ do not depend on $\omega$. By using Lemma (4), we know there exists a smooth Lyapunov function $V: \mathbb{R}^{n} \rightarrow \mathbb{R}_{\geq 0}$ for the system (5) $r$-homogeneous of degree $k$. As before, there exists a neighborhood $\Lambda^{\prime \prime}$ of $\omega_{0}$ in $\Lambda$ such that $\dot{V}_{\omega}(x)<0$ for all $(\omega, x) \in \Lambda^{\prime \prime} \times \mathcal{S}$. As $x \mapsto f(\omega, x)$ is $r$-homogeneous of degree $d(\omega)$ and $V$ is $r$-homogeneous of degree $k$, it follows that $\dot{V}_{\omega}(x)$ is $r$-homogeneous of degree $d(\omega)+k$ for all $\omega \in \Lambda^{\prime \prime}$ (see for instance [10], [11]). Thus $\dot{V}_{\omega}(x)<0$ for all $(\omega, x) \in \Lambda^{\prime \prime} \times \mathbb{R}^{n *}$ and $V$ is a Lyapunov function for the system (4) for all $\omega \in \Lambda^{\prime \prime}$.

Remark 7: Theorem 6 gives the existence of a neighborhood $\Lambda^{\prime}$ and not a precise specification of the knowledge of the domain of stability. Numerical techniques could be used to determine a more precise domain of stability. Nevertheless, when the degree of homogeneity of the system (4) depends on the constant weights $\left(r_{1}, \ldots, r_{n}\right) \in \mathbb{R}_{>0}^{n}$ for all $\omega \in \Lambda$, the knowledge of a Lyapunov function can be useful to specify the neighborhood $\Lambda^{\prime \prime}$.

For systems without any homogeneity property, we still have the following result:

Corollary 8: If the system (5) is globally asymptotically stable for $\omega_{0} \in \Lambda$, then there exists a compact set $\mathcal{A}$ and a neighborhood $\Lambda^{\prime}$ of $\omega_{0}$ in $\Lambda$ such that $\mathcal{A}$ is strictly positively invariant under $x \mapsto f(\omega, x)$ for all $\omega$ in $\Lambda^{\prime}$.

The next result is a corollary of Theorem 6 which deals with the problem of stabilization.

Corollary 9: Consider the control system (2) and the continuous function

$$
\begin{aligned}
& u: \Lambda \times \mathbb{R}^{n} \rightarrow \mathbb{R}^{m} \\
& (\omega, x) \quad \mapsto u(\omega, x) .
\end{aligned}
$$

Suppose that for every $\omega \in \Lambda$ the vector field $x \mapsto$ $f(x, u(\omega, x))$ is homogeneous and all Cauchy problems associated with the closed-loop system

$$
\dot{x}=f(x, u(\omega, x)), \quad x \in \mathbb{R}^{n}
$$

have unique forward solutions for all positive times. If the control system (2) is globally continuously stabilized under the feedback control $u\left(\omega_{0}, x\right)$ with $\omega_{0} \in \Lambda$, then there exists a neighborhood $\Lambda^{\prime}$ of $\omega_{0}$ in $\Lambda$ such that for all $\omega \in \Lambda^{\prime}$, the system (2) is globally continuously stabilized under the feedback control $u(\omega, x)$.

\section{APPliCATION TO FINITE TIME ROBUST STABILIZATION}

An application of the previous theory is in studying the global finite time robust stabilization of the chain of integrators (see [1, Proposition 8.1]). In this example, a nonlinear robust feedback control is derived from a linear one by using Corollary 9.

The chain of integrators is given by

$$
\left\{\begin{array}{l}
\dot{x}_{1}=x_{2} \\
\vdots \\
\dot{x}_{n-1}=x_{n} \\
\dot{x}_{n}=u
\end{array}\right.
$$

denoted in short by

$$
\dot{x}=\Gamma(x, u) \text {. }
$$

The following characterization of the homogeneity property of the chain of integrators will be useful.

Lemma 10: The system (7) is $r$-homogeneous of degree $d$ if and only if

$$
r_{i+1}=r_{i}+d, \quad 1 \leq i \leq n-1
$$

with $d \in\left(-\frac{r_{n}}{n-1}, r_{n}\right]$ and $u$ is $r$-homogeneous of degree $r_{n}+d$.

In [1, Proposition 8.1], a control algorithm is built for the chain of integrators (7). In the following, we extend this result to a robust feedback control in the sense that we obtain a sector margin for the choice of the control gain. Indeed, we built a closed-loop chain of integrators which is robust to perturbations of the feedback control gains. Moreover, a more precise specification of the domain of stability is given by using Corollary 9 and Lemma 10.

Proposition 11: Let $k=\left(k_{1}, \ldots, k_{n}\right) \in \mathbb{R}^{n}$ such that $C(k)$ is Hurwitz. There exists $\epsilon \in\left[1-\frac{1}{n-1}, 1\right)$ and $\epsilon_{i}>0$ for $1 \leq i \leq n$ such that for all

$$
\begin{aligned}
\alpha & \in(1-\epsilon, 1), \\
k_{i}^{\prime} & \in\left(k_{i}-\epsilon_{i}, k_{i}+\epsilon_{i}\right),
\end{aligned}
$$

the system (7) is globally finite time stabilizable under the continuous feedback control

$$
u\left(\alpha, k_{1}^{\prime}, \ldots, k_{n}^{\prime}, x\right)=-k_{1}^{\prime}\left\lfloor x_{1}\right\rceil^{\alpha_{1}}-\ldots-k_{n}^{\prime}\left\lfloor x_{n}\right\rceil^{\alpha_{n}}
$$

with

$$
\lfloor x\rceil^{\alpha}=|x|^{\alpha} \operatorname{sgn}(x), \quad x \in \mathbb{R}
$$

and

$$
\left\{\begin{array}{l}
\alpha_{i-1}=\frac{\alpha_{i} \alpha_{i+1}}{2 \alpha_{i+1}-\alpha_{i}}, \quad 2 \leq i \leq n \\
\alpha_{n}=\alpha \\
\alpha_{n+1}=1
\end{array}\right.
$$

Moreover, the closed-loop system

$$
\dot{x}=\Gamma\left(x, u\left(\alpha, k_{1}^{\prime}, \ldots, k_{n}^{\prime}, x\right)\right)
$$

is $\left(\frac{1}{\alpha_{1}}, \ldots, \frac{1}{\alpha_{n}}\right)$-homogeneous of degree $d=\frac{\alpha-1}{\alpha}$. 
Proof: Homogeneity property: With Lemma (10), it is easy to verify that the system (10) is homogeneous of degree $d$ if and only the the following inequalities hold

$$
-\frac{1}{\alpha(n-1)}<\frac{\alpha-1}{\alpha}<\frac{1}{\alpha}, \quad n>1 .
$$

So the system must satisfy

$$
0<1-\frac{1}{n-1}<\alpha<2
$$

which is equivalent to the fact that

$$
-\frac{1}{n-1}<d<\frac{1}{2}
$$

Asymptotic stability: It is well known that the system

$$
\dot{x}=\Gamma\left(x, u\left(1, k_{1} \ldots, k_{n}, x\right)\right)
$$

is globally asymptotically stable. Corollary 9 implies that there exists $(a, b) \in \mathbb{R}_{>0}^{2}$ with

$$
1-\frac{1}{n-1}<a<1<b<2
$$

and $\epsilon_{i}>0$ with $1 \leq i \leq n$ such that the system (10) is globally asymptotically stable for all $\alpha \in(a, b)$ and $k_{i}^{\prime} \in$ $\left(k_{i}-\epsilon_{i}, k_{i}+\epsilon_{i}\right)$.

Finite time convergence: It follows from [1, Theorem 7.1] that the closed-loop system (10) is finite time stable if the degree of homogeneity $d$ is negative. Finally, we have

$$
1-\frac{1}{n-1}<\alpha<1
$$

Remark 12: Due to the fact that the function $x \mapsto\lfloor x\rceil^{\alpha}$ is not differentiable for $0<\alpha<1$, the closed-loop system (10) is not equivalent to the closed-loop system

$$
\dot{x}=\Gamma\left(x, u\left(1, k_{1} \ldots, k_{n}, x\right)\right)
$$

or a well known stabilizable system under a diffeomorphic change of coordinates in the state space. This is the reason why we had to find a new way of doing.

Example 13: Let us give the example of the triple integrator

$$
\left\{\begin{array}{l}
\dot{x}_{1}=x_{2} \\
\dot{x}_{2}=x_{3} \\
\dot{x}_{3}=u
\end{array} .\right.
$$

For $\alpha=\frac{11}{20}, k_{1}=-1, k_{2}=k_{3}=-\frac{3}{2}$, the feedback control (8) becomes

$u\left(\frac{11}{20},-1,-\frac{3}{2},-\frac{3}{2}, x\right)=-\left\lfloor x_{1}\right\rfloor^{\frac{11}{38}}-\frac{3}{2}\left\lfloor x_{2}\right\rceil^{\frac{11}{29}}-\frac{3}{2}\left\lfloor x_{3}\right\rfloor^{\frac{11}{20}}$.

Then, the system is $\left(\frac{38}{11}, \frac{29}{11}, \frac{20}{11}\right)$-homogeneous of degree $-\frac{9}{11}$. A simulation of the system leads to Figure 1 which gives an estimate of the settling time.

With Proposition 11, we know that for $\alpha=\frac{1}{2}$ we lose the finite time property as displayed by Figure 2 .

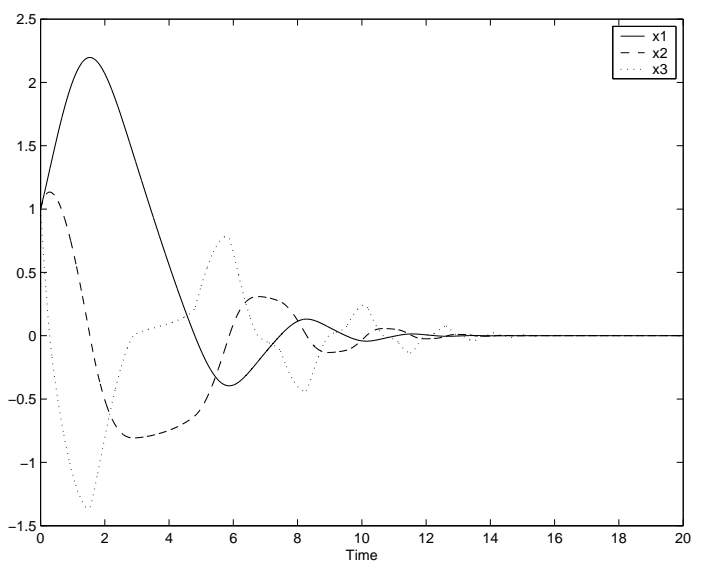

Fig. 1. Simulation with $\alpha=\frac{11}{20}$

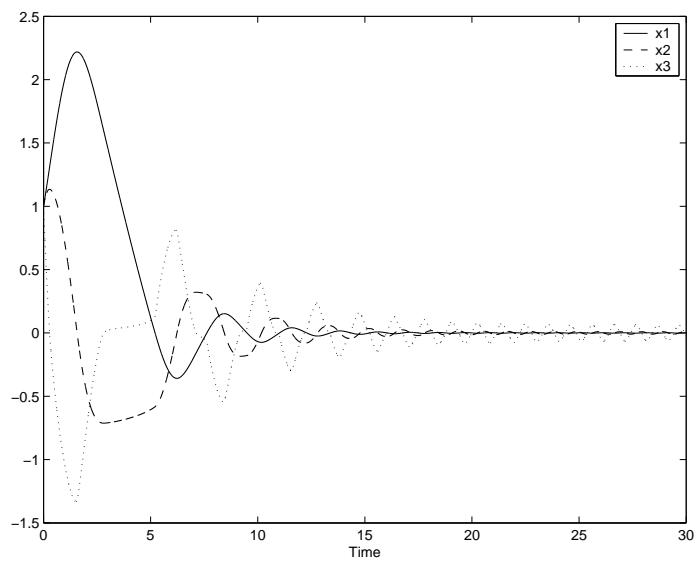

Fig. 2. Simulation with $\alpha=\frac{1}{2}$

\section{CONClusion}

This paper analyzes the stability and stabilization of systems depending on a parameter. The main goal of this paper is to develop a topological tool to obtain the global stability and stabilization of a system class depending on a parameter. The tube lemma plays a key role in the presented theoretical results. These are particularly suited for deriving a nonlinear feedback control from a linear one as for the chain of integrators.

A possible direction for future research is to build a state space varying parameter $\omega(x)$, with values in the neighborhood $\Lambda^{\prime}$, in order to optimize the properties of the feedback controls $u(\omega(x), x)$, as for instance the speed of convergence. It will be interesting to find a more precise specification of the neighborhood $\Lambda^{\prime}$, or at least of $\partial \Lambda^{\prime}$, in order to bound the state space varying parameter $\omega(x)$.

\section{ACKNOWLEDGMENT}

The author gratefully acknowledge discussions on homogeneous systems with Laurent Praly.

\section{REFERENCES}

[1] S. P. Bhat and D. S. Bernstein, "Geometric homogeneity with applications to finite-time stability," Math. Control Signals Systems, vol. 17, pp. 101-127, 2005. 
[2] E. Moulay and W. Perruquetti, Finite-time stability and stabilization: state of the art, in "Advances in Variable Structure and Sliding Mode Control". Lecture Notes in Control and Information Sciences, SpringerVerlag, 2006, vol. 334.

[3] — - "Finite time stability and stabilization of a class of continuous systems," J. Math. Anal. Appl., vol. 323, no. 2, pp. 1430-1443, 2006.

[4] L. P. Rothschild and E. M. Stein, "Hypoelliptic differential operators and nilpotent groups," Acta Math., vol. 137, pp. 247-320, 1976.

[5] N. Bhatia and O. Hajek, Local semi-dynamical systems. Lecture Notes in Mathematics, Vol. 90, Springer, 1969.

[6] S. P. Bhat and D. S. Bernstein, "Finite time stability of continuous autonomous systems," SIAM J. Control Optim., vol. 38, no. 3, pp. 751$766,2000$.

[7] L. Rosier, "Homogeneous Lyapunov function for homogeneous continuous vector field," Systems Control Lett., vol. 19, pp. 467-473, 1992.

[8] J. Munkres, Topology. Prentice Hall, 1999.

[9] J. Kurzweil, "On the inversion of Liapunov's second theorem on stability of motion," Amer. Math. Soc. Transl., vol. 24, pp. 19-77, 1963.

[10] H. Hermes, "Homogeneous ccordinates and continuous asymptotically stabilizing feedback controls," in Proc. Colorado Springs Conf., Differential equations : Stability \& Control,Marcel Dekker, S. Elaydi Editor, 1990.

[11] M. Kawski, "Geometric homogeneity and stabilization," in Proc. IFAC Nonlinear Control Symposium, Lake Tahoe, CA, 1995, pp. 164-169. 